\title{
THEORY FOR TEMPERATURE INDUCED MAGNETIC REORIENTATION IN THIN FILMS AND MULTILAYERS
}

\author{
P.J. JENSEN \\ Hahn-Meitner-Institut, Glienicker Str. 100, 14109 Berlin, Germany
}

The temperature driven reorientation of the magnetization observed in thin ferromagnetic films and multilayer systems is studied theoretically. We take into account layer dependent magnetic anisotropies. The free energy and the virection of magnetization are calculated by using a perturbational approach as well as a nonperturbational treatment. It is shown that in most cases a continuous magnetic reorientation is obtained. An increasing anisotropy energy as compared to the exchange interaction leads to an increasing width of the magnetic reorientation.

PACS numbers: 75.10.IIk, 75.30.Gw, 75.70.Ak

The reorientation of the magnetization of thin films and multilayers between perpendicular $\left(M_{\perp}\right)$ and in-plane direction $\left(M_{\|}\right)$has been studied intensively both experimentally [1-3] and theoretically [4-6]. With increasing temperature a reversible perpendicular to in-plane rotation $M_{\perp} \rightarrow M_{\|}$as well as the reversed reorientation $M_{\|} \rightarrow M_{\perp}$ was observed. In addition some systems show an in-plane (azimuthal) magnetic reorientation.

The magnetic reorientation is caused by the interplay of the dipolar interaction, the surface (or interface) anisotropy $\mathcal{K}_{\text {surf }}$, and the anisotropy contributions of the film interior ("volume") $\mathcal{K}_{\text {vol }}$ (bulk and strain anisotropies $[3,7]$ ). The diminished relative magnetization $m(T)$ causes effective, temperature dependent anisotropies, since with increasing temperatures their ability to align the magnetization decreases. Thus, the anisotropies are temperature dependent mainly through the magnetization [8]. Most interestingly, these effective anisotropies will be layer dependent, since for thin films the magnetization itself is layer dependent [9]. In general, with increasing temperature the magnetization decreases faster at the film surface than in the interior of the film. This leads to a faster decrease of the surface anisotropy as compared to the anisotropic contributions located in the film interior, resulting in a magnetic reorientation [10]. Consequently, the magnetic reorientation can be driven thermodynamically.

Dependent on the effective anisotropies ("anisotropy flow" [11]), the magnetic reorientation may occur discontinuously (step-like) as well as continuously. 
Whereas both orders of transitions are expected to occur, experimentally mainly smooth magnetic reorientations are observed $[1,2]$. In this study we investigate theoretically the existence, the location, and in particular the order of the reorientation transition in thin films with several atomic layers, dependent on the relative sign and strength of the anisotropies.

Consider a thin (001)-film wilh $N$ uniformly ordered atomic layers and classical vector spins $S_{i}\left(\left|S_{i}\right|=1\right)$ located on each lattice site $i$. In addition to the isotropic exchange coupling we consider the anisotropic IIamiltonian

$$
\mathcal{H}_{\text {anis }}=-\sum_{i} \mathcal{K}_{2, i}\left(S_{i}^{z}\right)^{2}+\frac{1}{2}\left(g \mu_{\mathrm{B}}\right)^{2} \sum_{\substack{i, j \\ i \neq j}}\left[\frac{S_{i} S_{j}}{r^{3}}-\frac{3}{r^{5}}\left(r S_{i}\right)\left(r S_{j}\right)\right]
$$

$\mathcal{K}_{2, i}$ is the second order uniaxial lattice anisotropy, varying for different film layers. In the following we put $\mathcal{K}_{2 \text {,surf }}$ for the two surface/interface layers, and $\mathcal{K}_{2 \text {,vol }}$ for all interior film layers. Higher order lattice anisotropies are neglected. The long range dipole interaction couples spins on lattice sites $i$ and $j, r=r_{i}-r_{j}, g$ is the Landé factor, and $\mu_{\mathrm{B}}$ the Bohr magneton. The quantization axis is determined by the angle 0 between the magnetization and the film normal $(\theta=0$ perpendicular, $\theta=\pi / 2$ in-plane magnetization). Due to the strong exchange coupling $J$ a collinear ordering is assumed, therefore 0 is equal for all film layers.

The relative magnetization $m_{i}(T)=m_{\alpha}(T)$ of film layer $\alpha$ at temperature $T$ is calculated within the molecular field approximation, which is given by the Langevin function for an isotropic system [9]

$$
\begin{aligned}
& m_{\alpha}(T)=\operatorname{coth}_{1}\left(H_{\alpha}^{\mathrm{mf}} / k_{\mathrm{B}} T\right)-k_{\mathrm{B}} T / H_{\alpha}^{\mathrm{mf}}, \\
& H_{\alpha}^{\mathrm{mf}}=J\left(z_{0} m_{\alpha}+z_{1} m_{\alpha+1}+z_{1} m_{\alpha-1}\right) .
\end{aligned}
$$

$H^{\mathrm{mf}}$ is the molecular field, $z_{0}$ and $z_{1}$ are the numbers of nearest neighbors in the same layer and between adjacent layers, and $m_{0}=m_{N+1}=0$.

First, a thermodynamic perturbational expansion of the free energy (cumulant expansion) to linear order of the anisotropy couplings is applied [8], yielding

$$
\begin{gathered}
F(T, \theta) \approx F_{0}(T)-\sum_{i} \mathcal{K}_{2, i}\left[1-3 \frac{k_{\mathrm{B}} T}{H_{i}^{\mathrm{mf}}} \operatorname{coth}\left(\frac{H_{i}^{\mathrm{mf}}}{k_{\mathrm{B}} T}\right)+3\left(\frac{k_{\mathrm{B}} T}{H_{i}^{\mathrm{mf}}}\right)^{2}\right] \cos ^{2} \theta \\
+\frac{1}{2}\left(g \mu_{\mathrm{B}}\right)^{2} \sum_{\substack{i, j \\
i \neq j}}\left[\frac{m_{i}(T) m_{j}(T)}{r^{3}}-\frac{3}{r^{5}}\left(r m_{i}(T)\right)\left(r m_{j}(T)\right)\right] .
\end{gathered}
$$

Here, $m_{i}=m_{i}(\sin \theta, 0, \cos \theta)$, and $F_{0}(T)$ is the free energy of the isotropic Hamiltonian. The second term denotes the effective, temperature dependent anisotropy $\mathcal{K}_{2, i} \equiv \mathcal{K}_{2, \alpha}$ of film layer $\alpha . \mathcal{K}_{2, \alpha}(T)$ is temperature dependent mainly through the magnetization $m_{\alpha}(T)$, therefore $\mathcal{K}_{2, \alpha}(T) \rightarrow 0$ for $T \rightarrow T_{\mathrm{c}}{ }^{\dagger}$. Since only second order anisotropies and the linear perturbational expansion are taken into account

tWe emphasize that only the effective anisotropy $\mathcal{K}_{2, \alpha}(T)$ vanishes for $T \rightarrow T_{c}$. We assume that the underlying spin-orbit coupling and the resulting lattice anisotropy $\mathcal{K}_{2, \alpha}$ stay constant in the considered temperature range. 
here, only the two solutions $\theta=0$ and $\theta=\pi / 2$ are stable. Thus, within this approximation one obtains a discontinuous magnetic reorientation only.

Secondly, as was shown previously by Moschel and Usadel [6], the application of a nonperturbative approach results in a continuous transition, despite only second order anisotropies are taken into account. Then a canted thin film magnetization occurs in a finite temperature range. This can be seen already from the quadratic perturbational expansion term of $F(T, 0)$, leading to the appearance of higher order anisotropy contributions at finite temperatures, and thus to the possibility of a smooth transition. Therefore, we analyze the behavior of the magnetic reorientation also with a nonperturbative calculation of the free energy $F(T, \theta)$.

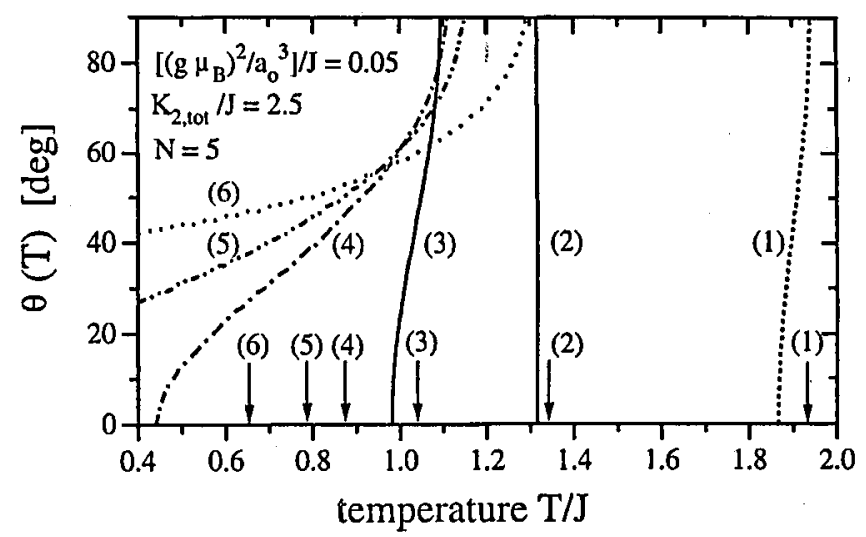

Fig. 1. Perpendicular to in-plane magnetic reorientation as a function of temperature for different ratios of $\mathcal{K}_{2, \text { surf }}$ and $\mathcal{K}_{2, \text { vol }}$. A fcc (001) film with $N=5$ atomic layers is assumed. The temperature and the anisotropies are given in units of the exchange conpling $J$. The curves are calculated by the nonperturbative treatment and refer to different values of $\mathcal{K}_{2 \text {,surf }}$ and $\mathcal{K}_{2 \text {,vol }}:(1) \mathcal{K}_{2, \text { surf }} / J=0.2, \mathcal{K}_{2, \text { vol }} / J=0.7 ;(2) 0.5,0.5$; (3) $0.8,0.3$; (4) $1.1,0.1$; (5) $1.25,0.0$; (6) $1.70,-0.3$. A step-like reorientation is ob-

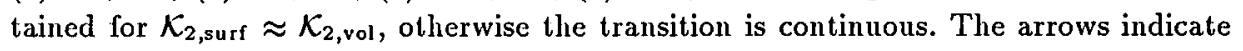
the respective reorientation tempcratures $T_{R}$ as calculated by the linear perturbational expansion, Eq. (1).

We have calculated the magnetic reorientation for different thin film systems. In Fig. 1 the behavior of the equilibrium angle $O(T)$ is shown for different values of $\mathcal{K}_{2 \text {,surf }}, \mathcal{K}_{2, \text { vol }}$, and a constant total lattice anisotropy $2 \mathcal{K}_{2 \text {,surf }}+(\mathrm{N}-2) K_{2 \text {,vol }}$.

In Fig. 2 the magnetic reorientation is shown for varying ratios of the exchange coupling and the anisotropies, whereas the ratios between the different anisotropic contributions stay constant. The results may be summarized as follows:

(i) In almost all investigated cases the obtained reorientation is smooth, i.e. a canted magnetization occurs in an finite temperature range. A step-like reorientation is obtained only for $\mathcal{K}_{2 \text {,surf }} \approx \mathcal{K}_{2, \text { vol }}$, i.e. for a uniform distribution of the lattice anisotropy, as has been pointed out already by Moschel et al. [6]. The resulting reorientation temperature $T_{\mathrm{R}}$ increases for increasing $\mathcal{K}_{2 \text {,vol }}$, cf. Fig. 1. 


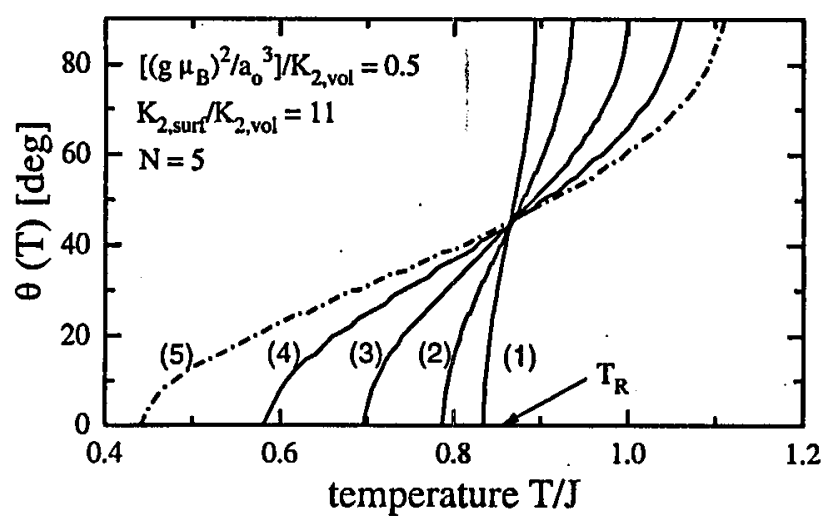

Fig. 2. Perpendicular to in-plane magnetic reorientation as a function of temperature for different ratios of the exchange coupling and the anisotropy interactions. Here the mutual ratios of the anisotropy contributions are assumed to be the same: $\mathcal{K}_{2 \text {,surf }} / \mathcal{K}_{2, \text { vol }}=11,\left[\left(g \mu_{\mathrm{B}}\right)^{2} / a_{0}^{3}\right] / \mathcal{K}_{2, \text { vol }}=2$. The curves as calculated nonperturbatively refer to: (1) $J / \mathcal{K}_{2, \mathrm{vol}}=100$; (2) 75 ; (3) 50 ; (4) 25 ; (5) 10 . All five curves for $\theta(T)$ merge into a single point, whose temperature is given by the reorientation temperature $T_{\mathrm{R}}$ as obtained from Eq. (4). For the other notations we refer to Fig. 1.

(ii) For constant mutual ratios of the anisotropies, cf. Fig. 2, the range of the reorientation decreases strongly upon decreasing the strength of the anisotropies relative to the exchange coupling $J$, whereas $T_{\mathrm{R}}$ does not change significantly.

(iii) The reorientation temperature $T_{\mathrm{R}}$ as obtained from the first order perturbation theory is located within the range of transition as calculated nonperturbatively. Thus, the former calculation allows for a fast determination of $T_{\mathrm{R}}$, which can then be used to analyze the magnetic reorientation in greater detail with the much more elaborate nonperturbative treatment.

Furthermore, the cases investigated here refer to a perpendicular to in-plane magnetic reorientation with increasing temperature. For $\mathcal{K}_{2 \text {,surf }}<0$ and $\mathcal{K}_{2, \text { vol }}>0$ also the reversed transition may occur. For strongly differing surface and volume anisotropies a noncollinear thin film magnetization is expected [6], characterized by a layer depending angle $\theta_{\alpha}(T)$. The inclusion of higher order uniaxial lattice anisotropies should also influence the location and order of the magnetic reorientation. A nonvanishing in-plane anisotropy $\mathcal{K}_{4, \alpha}^{\|}$with different signs of $\mathcal{K}_{4, \text { surf }}^{\|}$and $\mathcal{K}_{4, \text { vol }}^{\|}$may induce an in-plane (azimuthal) continuous magnetic reorientation.

\section{References}

[1] D.P. Pappas, K.P. Kämper, H. Hopster, Phys. Rev. Lett. 64, 3189 (1990); R. Allenspach, A. Bischof, Phys. Rev. Lett. 69, 3385 (1992); Z.Q. Qiu, J. Pearson, S.D. Bader, Phys. Rev. Lett. 70, 1006 (1993).

[2] H. Fritzsche, J. Kollhepp, H.J. Elmers, U. Gradmann, Phys. Rev. B 49, 15665 (1994); O. Schulte, F. Klose, W. Felsch, Phys. Rev. B 52, 6480 (1995). 
[3] F. IIuang, M.T. Kief, G.J. Mankey, R.F. Willis, Phys. Rev. B 49, 3962 (1994); B. Schulz, K. Baberschke, Phys. Rev. B 50, 13467 (1994).

[4] D. Pescia, V.L. Pokrovsky, Phys. Rev. Lett. 65, 2599 (1990); A. Hucht, A. Moschel, K.D. Usadel, J. Magn. Magn. Mater, 148, 32 (1995).

[5] P.J. Jensen, K.H. Bennemann, Phys. Rev. B 42, 849 (1990); Solid State Commun. 83, 1057 (1992); D.L. Mills, J. Magn. Magn. Mater. 100, 515 (1991); D.K. Morr, P.J. Jensen, K.II. Bennemann, Surf. Sci. 307-309, 1109 (1994).

[6] A. Moschel, K.D. Usadel, Phys. Rev. B 49, 12868 (1994); Phys. Rev. B 51, 16111 (1995).

[7] E. du Trémolet de Lacheisserie, Phys. Rev. B 51, 15925 (1995).

[8] H.B. Callen, E. R. Callen, J. Phys. Chem. Solids 27, 1271 (1966); P.J. Jensen, K.H. Bennemann, Ann. Physik 2, 475 (1993); Y. Millev, M. Fähnle, J. Magn. Magn. Mater. 135, 343 (1994); Phys. Rev. B 51, 2937 (1995).

[9] P.J. Jensen, H. Dreyssé, K.H. Bennemann, Europhys. Lelt. 18, 463 (1992); Surf. Sci. 269/270, 627 (1992).

[10] P.J. Jensen, K.H. Bennemann, submitted to Solid State Commun.

[11] P.J. Jensen, K.H. Bennemann, Phys. Rev. B 52, 16012 (1995); Y. Millev, J. Kirschner, preprint (1996). 